\title{
Acute Effects of Oscillatory PEP and Thoracic Compression on Secretion Removal and Impedance of the Respiratory System in Non- Cystic Fibrosis Bronchiectasis
}

\author{
Letícia Helena de Souza Simoni, Daniele Oliveira dos Santos, Hugo Celso Dutra de Souza, \\ José Antonio Baddini-Martinez, Marcel Koenigkam Santos, and Ada Clarice Gastaldi
}

\begin{abstract}
BACKGROUND: Bronchiectasis is characterized by abnormal and permanent dilatation of the bronchi, caused mainly by the progression of inflammatory processes and loss of the ability to remove mucus. Techniques to clear the airways are essential for the treatment of these patients. In this study, we aimed to evaluate the acute effects of oscillatory PEP and thoracic compression on both the clearance of secretions and impedance of airways in subjects with bronchiectasis. METHODS: This was a randomized crossover single-blinded study that involved both subjects with bronchiectasis and healthy subjects evaluated by using an impulse oscillometry system, which assessed resistance at $5 \mathrm{~Hz}$ and resistance $20 \mathrm{~Hz}$, reactance at $5 \mathrm{~Hz}$, reactance area, and resonant frequency, before, after, and $30 \mathrm{~min}$ after oscillatory PEP, chest compression, or control sessions. Dry and total weights, adhesiveness, purulence of the expectorated secretions, the dyspnea scale score, the acceptability and tolerance scale score, pulse oximetry, and difficulty in expectoration were also assessed. RESULTS: The dry and total weights of secretions were higher after the use of the oscillatory PEP technique than those in a control session $(P=.005$ and $P=.039$, respectively). In the bronchiectasis group, there was a decrease after oscillatory PEP in total airway resistance $(P=.04)$, peripheral resistance $(P=.005)$, and reactance area $(P=.001)$. After compression, there was a decrease in peripheral resistance $\mathrm{Hz}(P=.001)$ and reactance area $(P=.001)$. In the healthy group, there was an increase in resistance at $5 \mathrm{~Hz}(P=.02)$ after oscillatory PEP. There were no differences in acceptability and tolerance, dyspnea, and oxygen saturation. CONCLUSIONS: The oscillatory PEP technique was effective for the removal of secretions and in decreasing total and peripheral respiratory system resistance; thoracic compression had comparable positive effects on the peripheral resistance. Both techniques were safe and well tolerated by the subjects with bronchiectasis. ClinicalTrials.gov registration NCT02509637.) Key words: impulse oscillometry; pulmonary function; bronchiectasis; chest physiotherapy. [Respir Care 2019;64(7):818-827. (C) 2019 Daedalus Enterprises]
\end{abstract}

\section{Introduction}

Bronchiectasis is characterized on pathologic examination by the abnormal and permanent dilatation of one or

\footnotetext{
Ms de Souza Simoni, Ms dos Santos, Dr de Souza, and Dr Gastaldi are affiliated with the Department of Health Science, University of São Paulo, Ribeirão Preto, Brazil. Drs Baddini-Martinez and Santos are affiliated with the Internal Medicine Department, Ribeirão Preto Medical School, University of São Paulo, Ribeirão Preto, Brazil.
}

Financial support was provided by São Paulo Research Foundation (2013/ 26433-0 process). more bronchi with core symptoms, including chronic cough, expectoration of purulent secretions, and dyspnea. ${ }^{1-3}$ Furthermore, recurrent and chronic damage to the airways leads to a vicious cycle of bacterial colonization, inflammation, and tissue destruction. ${ }^{2,4-7}$ Other than pharmaco-

\footnotetext{
Ms de Souza Simoni presented a summary of the article at the XXXVIII Brazilian Congress of Pulmonology and Tisiology, held October 11-15, 2016, in Rio de Janeiro, Brazil. Dr Gastaldi presented a summary of the article at the World Confederation for Physical Therapy, held July 2017, in Cape Town, South Africa.
} 


\section{Oscillatory PEP and Thoracic COMPREsSion IN Non-CF BronchiECTASIS}

logic or surgical treatment, physical therapy techniques used to clear the airways are also indicated for patients with bronchiectasis; these techniques are essential in the treatment protocol and improve sputum expectoration, respiratory symptoms, and quality of life of these individuals. ${ }^{8,9}$

Different airway clearance techniques are available: either manual techniques or techniques performed by using specific devices. One of these techniques is manual thoracic compression, which requires the actions of a therapist during the expiratory phase to increase the expiratory flow. ${ }^{10-13}$ Although thoracic compression is a well-known technique and has been used for many years, the effects of this technique on the removal of secretions are not well defined. The sudden application of compression can lead to bronchial collapse in patients with COPD, as evaluated by peak flow measurements, ${ }^{14}$ and we do not know if this could also happen in patients with bronchiectasis when compression is applied during a fast or a slow maneuver. Another technique, which uses a device known as the FLUTTER, was proposed by Lindeman ${ }^{15}$ in 1992 and has been used frequently since that time. ${ }^{16}$ This technique is based on 2 principles: positive pressure combined with oscillatory flow during expiration..$^{15,17-20}$

The most frequently used method for assessing pulmonary function is spirometry. Other methods, for example, the impulse oscillometry system, have been proposed more recently. ${ }^{21,22}$ The impulse oscillometry system measures respiratory system impedance, which is a functional evaluation of the airways in response to pressure and flow variations. In addition to the ease of use, the system is more sensitive to alterations related to the central and peripheral airways, which may not be possible with spirometry. ${ }^{22}$ Our study evaluated the acute effects of oscillatory PEP and thoracic compression on secretion and impedance of airways in subjects with bronchiectasis and in healthy subjects.

\section{Methods}

\section{Design}

This study was a randomized crossover single-blinded study.

\footnotetext{
Correspondence: Ada Clarice Gastaldi PhD, Department of Health Science, Ribeiraõ Preto Medical School, University of São Paulo, Bandeirantes Avenue, 3900-14049-900 - Ribeirao Preto, Brazil. E-mail: ada@fmrp.usp.br.
}

DOI: $10.4187 /$ respcare.06025

\section{QUICK LOOK}

\section{Current knowledge}

For patients with bronchiectasis, airway clearance techniques are an essential aspect of treatment for secretion retention. The effect of the oscillatory PEP device on respiratory system resistance in individuals with chronic respiratory disease needs to be better defined.

\section{What this paper contributes to our knowledge}

The oscillatory PEP was effective for removal of secretions and to decrease total respiratory system resistance. Oscillatory PEP and thoracic compression had comparable positive effects on peripheral resistance and were safe and well tolerated by the subjects with bronchiectasis.

\section{Subjects}

To create the bronchiectasis group, we recruited volunteers who were in clinical follow-up at the pulmonology out-patient clinic of the Hospital das Clínicas of the Medical School of Ribeirão Preto. To be considered eligible for the study, subjects ages 30-80 y; diagnosed with bronchiectasis according to the bronchiectasis severity index, ${ }^{23}$ based on clinical history and high-definition computed tomography; and showed no exacerbation in the 4 weeks before the study were included in the study. Patients were excluded if they had COPD, asthma, other restrictive disease, a history of pulmonary surgery, or other pulmonary conditions. In the healthy group, the volunteers were matched by age, with normal spirometry findings and no history of respiratory or cardiac problems, and were recruited in the community. The study was approved by the human research ethics committee of the Clinical Hospital of the Ribeirão Preto Medical School, University of São Paulo.

\section{Intervention}

The subjects selected to participate in the study underwent a detailed medical evaluation to assess their general state of health and clinical stability. They were informed about all stages of the research, signed a consent form, and were instructed to suspend the use of inhaled respiratory medications $24 \mathrm{~h}$ before the study. The evaluations were performed in the Laboratory of Respiratory Assessment, Physical Therapy Graduation Course, Ribeirão Preto Medical School, University of São Paulo. At baseline, all the subjects underwent complete lung function testing, with residual volume measurement, sputum bacteriology, and 


\section{Oscillatory PEP AND Thoracic COMPRESSION IN Non-CF BRONCHIECTASIS}

dyspnea scale evaluation by using the Medical Research Council scale. ${ }^{24}$ The expectoration difficulty scale was also applied..$^{25}$ The scale ranges from 1 to $5: 1$ is no difficulty and 5 is extremely difficult. The bronchiectasis severity index was also assessed. ${ }^{23}$ The bronchiectasis severity index is a new index for classification of the severity of bronchiectasis according to both clinical and radiologic criteria.

During each session, the subjects, in a seated position, were instructed to perform 3 forced expirations every $5 \mathrm{~min}$ to eliminate secretions. The secretions were evaluated for their weight, adhesiveness, and purulence. During the control session, the subjects remained seated and breathed calmly with no guidance; in the Flutter VRP1 (VarioRaw SA, Switzerland) session, the subjects performed exercises with calm inspiration and prolonged expiration; and, in the compression session, the subjects were instructed to take deep breaths, interspersed with 3 quiet breaths and the expirations were accompanied by bilateral slow compression with the therapist's hands (LHSS) on the lower ribs. In the oscillatory PEP and compression sessions, 1-min rests were planned every $4 \mathrm{~min}$.

The control, oscillatory PEP, or compression sessions were randomly assigned by using Microsoft Excel (Microsoft, Redmond, WA). The techniques were applied by the same experienced physiotherapist (LHSS), and another investigator (DOS) performed the impulse oscillometry system tests, blinded to the sessions. The study was conducted on $3 \mathrm{~d}$ with 1 week of washout. Each session consisted of baseline evaluation (pre); $30 \mathrm{~min}$ of control, oscillatory PEP, or compression session; immediate evaluation (post); and $30 \mathrm{~min}$ of rest and re-evaluation (rest).

\section{Primary Outcomes}

Secretions. The secretions were collected in universal weighing scales for total and dry weight evaluation. They were also evaluated for adhesiveness according to the visual scale described by Lopez-Vidriero, ${ }^{26}$ which ranges from 1 (more adhesive) to 4 (less adhesive). The purulence was evaluated as either mucoid, mucopurulent, or purulent according to the visual scale proposed by Murray et al. ${ }^{27}$

Impulse Oscillometry. Impulse oscillometry was performed by using a Jaeger impulse oscillometry system (Jaeger, Wurzburg, Germany) calibrated daily for volume and pressure by using a 3-L syringe with a reference resistance of $0.2 \mathrm{kPa} / \mathrm{L} / \mathrm{s}$. A free-flow mouthpiece was used to minimize the effect of malposition of the tongue. ${ }^{28}$ The mouthpiece contains a depressor to keep the tongue on the floor of the mouth, stabilize it, and reduce oral resistance. Pressure pulses were applied to the airways 5 times per second for at least $60 \mathrm{~s}$ while the subject was breathing at the tidal volume level. The pulses were performed to calculate the mean of 3 technically acceptable measurements. Parameters were calculated by using frequencies between 5 and $35 \mathrm{~Hz}$, including the resistance at $5 \mathrm{~Hz}$ (R5) or total respiratory system resistance; resistance at $20 \mathrm{~Hz}$ (R20) or central resistance; R5-R20 or peripheral resistance; reactance at $5 \mathrm{~Hz}$, which reflects the combined effect of tissue elastance and inertance; reactance area; and resonant frequency, the frequency at which the values of elastic and inertial resistance are equal. ${ }^{29,30}$

\section{Secondary Outcomes}

Acceptability and Tolerance. Thirty minutes after the treatment or control session, the subjects rated the procedure's utility, ease of understanding instructions, ease of performing the exercises, the degree of fatigue, and discomfort on scales of 1 to 7 (1, extremely; 7 , none). ${ }^{31}$

Pulse Oximetry. During the interventions, the subjects were monitored with a digital oximeter TuffSat (GE Healthcare, Helsinki, Finland), and the data from both before and after the procedure were recorded.

\section{Data Analysis}

The sample size was calculated by using previous results with the variable $\mathrm{R} 5$, when considering a mean $\pm \mathrm{SD}$ difference of $0.08 \pm 0.07^{21}$ power of $90 \%$, and $\alpha$ of $5 \%$, which resulted in a total of 14 individuals with a diagnosis of bronchiectasis (bronchiectasis group) and 14 healthy individuals (control group). Data were analyzed by using the statistical programs R (RCore Team, Vienna, Austria) and SAS Statistical Software (version 9.3, SAS Institute, Cary, North Carolina). Both were used to generate linear analysis models, with mixed effects. Data are presented as mean $\pm \mathrm{SD}$, and $95 \%$ CI. $P<.05$ was considered to be significant.

\section{Results}

Anthropometric and sociodemographic characteristics of the volunteers are shown in Table 1. Eighty-seven patients were screened, and $47 \mathrm{did}$ not meet the inclusion criteria, therefore, 40 subjects completed the protocol and were divided into the bronchiectasis and control groups (Fig. 1). Twenty subjects with bronchiectasis were evaluated; 14 expectorated secretions during the application of at least one of the techniques. Non-cystic fibrosis bronchiectasis etiology was as follows: tuberculosis sequelae $(40 \%)$, repeated childhood infection (30\%), ciliary dyskinesia (10\%), $\alpha-1$ antitrypsin deficiency (5\%), secondary to Jogren syndrome (5\%), immunoglobulin M deficiency (5\%), and non- 


\section{Oscillatory PEP and Thoracic COMPREsSion IN Non-CF BronchiECTASIS}

Table 1. Baseline Characteristics of the Study Participants

\begin{tabular}{|c|c|c|}
\hline Characteristic & $\begin{array}{l}\text { Subjects With } \\
\text { Bronchiectasis } \\
\quad(n=20)\end{array}$ & $\begin{array}{l}\text { Healthy } \\
\text { Subjects } \\
(n=20)\end{array}$ \\
\hline Age, mean $\pm \mathrm{SD}$ y & $57 \pm 14$ & $56 \pm 10$ \\
\hline $\mathrm{BMI}$, mean $\pm \mathrm{SD} \mathrm{kg} / \mathrm{m}^{2}$ & $24 \pm 4.6$ & $24 \pm 8$ \\
\hline \multicolumn{3}{|l|}{ Airway pathogens, $\%$} \\
\hline Pseudomonas aeruginosa & 25 & NA \\
\hline Staphylococcus aureus & 5 & NA \\
\hline No colonization & 50 & NA \\
\hline No sputum collection & 20 & NA \\
\hline MRC & 3 & 1 \\
\hline Difficulty of sputum & 2 & NA \\
\hline BSI, mean & 6 & NA \\
\hline $\mathrm{FEV}_{1}$, mean $\pm \mathrm{SD} \%$ pred. & $60.4 \pm 0.28$ & $103.5 \pm 13.8$ \\
\hline FVC, mean \pm SD $\%$ pred. & $79.4 \pm 0.24$ & $107.5 \pm 15.2$ \\
\hline $\mathrm{FEV}_{1} / \mathrm{FVC}$, mean $\pm \mathrm{SD} \%$ pred. & $62.7 \pm 0.17$ & $96.8 \pm 6.5$ \\
\hline $\mathrm{VC}$, mean $\pm \mathrm{SD} \%$ pred. & $78.6 \pm 0.24$ & NA \\
\hline $\mathrm{RV}$, mean $\pm \mathrm{SD} \%$ pred. & $112.8 \pm 0.21$ & NA \\
\hline TLC, mean \pm SD $\%$ pred. & $90.9 \pm 0.16$ & NA \\
\hline $\mathrm{RV} / \mathrm{TLC}$, mean $\pm \mathrm{SD} \%$ pred. & $125.5 \pm 0.25$ & NA \\
\hline $\begin{array}{l}\text { BMI }=\text { body mass index } \\
\text { NA = not applicable } \\
\text { MRC = Medical Research Council } \\
\text { BSI = bronchiectasis severity index } \\
\% \text { pred. = percent predicted } \\
\text { VC = vital capacity } \\
\text { RV = residual volume } \\
\text { TLC = total lung capacity }\end{array}$ & & \\
\hline
\end{tabular}

identified (5\%). The control group was made up of 20 healthy non-smokers with normal spirometry and in a similar age range to those who completed the protocol. Significant differences were found between oscillatory PEP and the control sessions for analysis of secretion removal by total weight $(P=.039)$ and dry weight $(P=.005)$ (Fig. $2)$. The average adhesion score was 2 in the oscillatory PEP and control sessions and was 3 in the compression session, but the differences were not significant. In all 3 interventions, the expectorated secretions were mucopurulent.

Results obtained by the impulse oscillometry system in the bronchiectasis group are shown in Table 2. No difference was found immediately after application of the techniques. In comparison between the post and rest. Significant changes were observed in the values of R5-R20 and $\mathrm{AX}$, after the oscillatory PEP session and thoracic compression, in the comparison with rest. When comparing pre versus rest and post versus rest evaluation time frames, both oscillatory PEP and thoracic compression produced a decrease in $\mathrm{R} 5-\mathrm{R} 20(\mathrm{kPa} / \mathrm{L} / \mathrm{s})$ and reactance area $(\mathrm{kPa} / \mathrm{L})$. In the control session, R5-R20 (kPa/L/s) parameter was increased during rest compared with the post evaluation. In the comparisons between the baseline evaluation versus re-evaluation and the immediate evaluation versus re-eval- uation, an increased reactance area $(\mathrm{kPa} / \mathrm{L})$ was observed. There was no difference for R20 in any comparison. The differences observed for parameters reactance at $5 \mathrm{~Hz}$ $(\mathrm{kPa} / \mathrm{L} / \mathrm{s})$ and resonant frequency $(\mathrm{Hz})$ were similar in both techniques and the control session. The values obtained in the impulse oscillometry system for the healthy group are shown in Table 3. After the oscillatory PEP intervention, there was an increase in $\mathrm{R} 5(\mathrm{kPa} / \mathrm{L} / \mathrm{s})$ and in $\mathrm{R} 5-\mathrm{R} 20$ $(\mathrm{kPa} / \mathrm{L} / \mathrm{s})$ when comparing the pre to the post or rest results, without a drop in these values after the re-evaluation period. When comparing post to rest data, thoracic compression showed a reduction in reactance at $5 \mathrm{~Hz}(\mathrm{kPa} / \mathrm{L} / \mathrm{s})$ and the control session exhibited an increase in reactance area $(\mathrm{kPa} / \mathrm{L})$. There was no difference for $\mathrm{R} 20$ in any comparison, and the other parameters showed similar results.

During the 3 interventions in both groups, there was no significant variation in oxygen saturation values, which ranged from $94.3 \pm 2.6 \%$ to $93.7 \pm 3.9 \%$ for the oscillatory PEP device, $94.2 \pm 3.0 \%$ to $93.9 \pm 31 \%$ for thoracic compression and $94.3 \pm 2.4 \%$ to $93.4 \pm 2.8 \%$ for the control intervention in the bronchiectasis group. In the control group, the variations were $97.1 \pm 1.6 \%$ to $97.1 \pm 2 \%$ for the oscillatory PEP intervention, $97.5 \pm 0.9 \%$ to $97 \pm 1.7 \%$ for thoracic compression, and $96.7 \pm 1.6 \%$ to $97.2 \pm 1.5 \%$ in the control intervention. The heart rate of the subjects also remained unchanged, which ranged from $80.1 \pm 134$ beats/min to $78.2 \pm 12.8$ beats/min for the oscillatory PEP intervention, $81.7 \pm 13.2$ beats $/ \mathrm{min}$ to $79.9 \pm 14.3$ beats $/ \mathrm{min}$ for thoracic compression, and $83.1 \pm 14.2$ beats/min to $81.6 \pm$ 12.0 beats/min for the control intervention in the bronchiectasis group. In the control group, the variations were $75.4 \pm 10.1$ beats/min to $73.5 \pm 9.1$ beats/min for the oscillatory PEP intervention, $73.7 \pm 7.6$ beats $/ \mathrm{min}$ to $73.5 \pm 9.6$ beats $/ \mathrm{min}$ for thoracic compression and $73.3 \pm 7.3$ beats $/ \mathrm{min}$ to $73.2 \pm 5$ beats $/ \mathrm{min}$ for the control intervention. With regard to the scales of acceptability and tolerance, both interventions and the control session were well accepted by the participants, with better scores for acceptability and tolerance being assigned most frequently (in $>70 \%$ of cases). Only oscillatory PEP in the bronchiectasis group received a score of $40 \%$, which reflected the degree of fatigue experienced (exhaustion) (Table 4).

\section{Discussion}

To our knowledge, this research was the first study that evaluated the acute effects of oscillatory PEP and thoracic compression techniques on respiratory system resistance in subjects with non-cystic fibrosis bronchiectasis. Only the oscillatory PEP technique was effective for secretion removal and had a beneficial effect on the total and peripheral respiratory system resistance, whereas thoracic compression decreased the peripheral resistance in subjects 


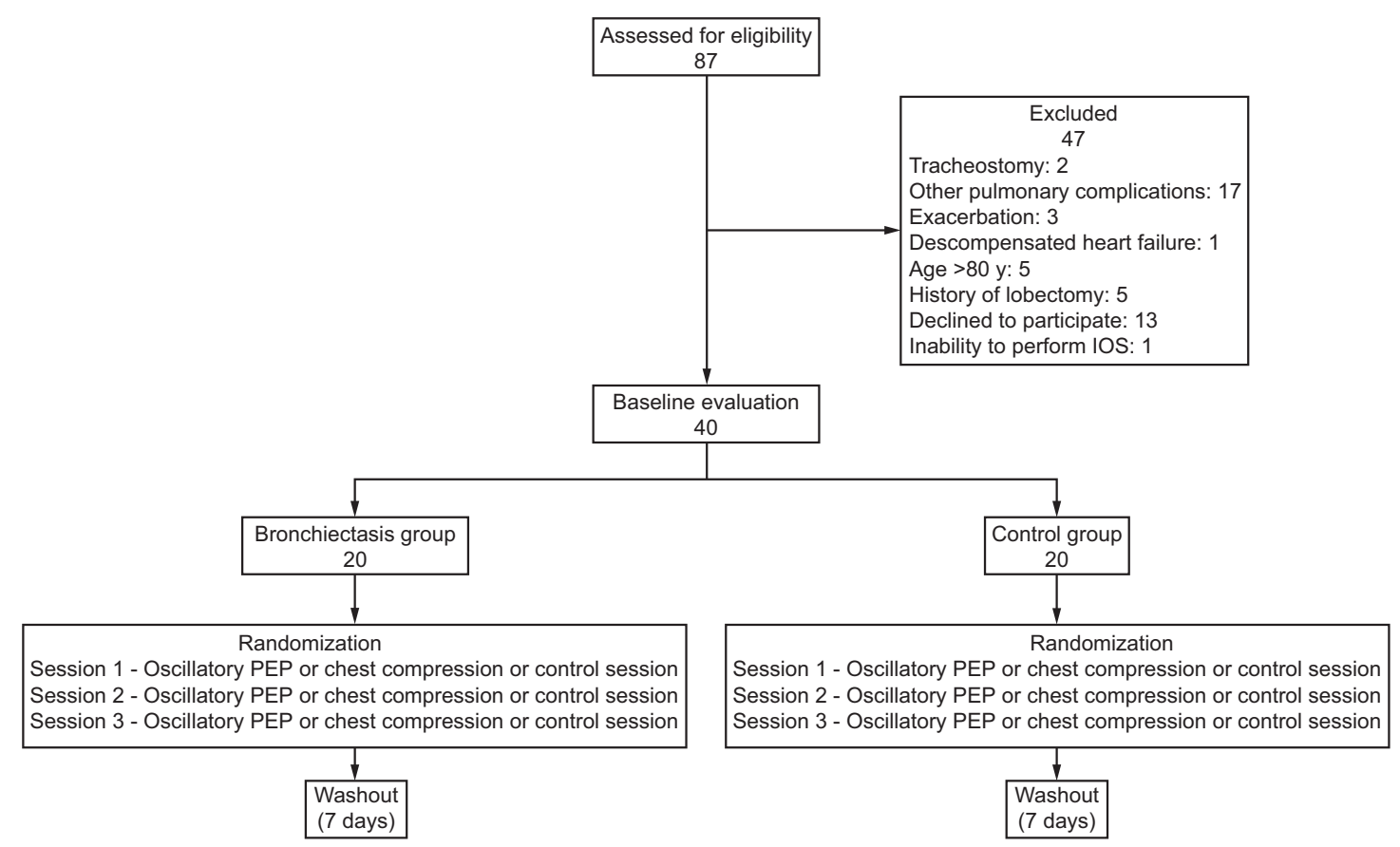

Fig. 1. Eligibility, randomization, and interventions.
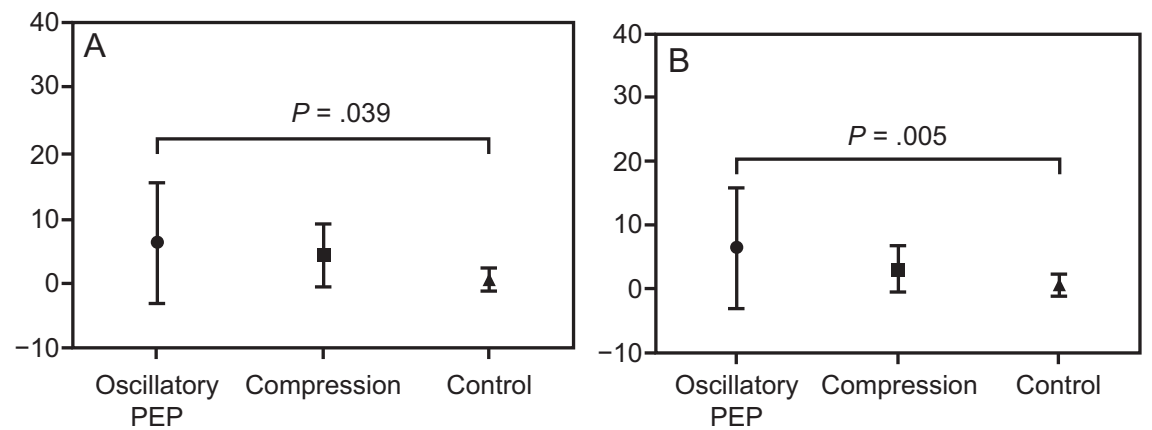

Fig. 2. Total weight $(A)$ and dry weight $(B)$ of the secretion expectorated during oscillatory PEP, compression, and control sessions in the bronchiectasis group.

with bronchiectasis. However, oscillatory PEP produced an increase in resistance in the healthy subjects. There was no decrease in saturation or change in the heart rate during either intervention. The study participants stated that the techniques were well accepted and did not generate discomfort.

According to the bronchiectasis severity index, our group of subjects had moderate bronchiectasis. To the best of our knowledge, no other physical therapy study on subjects with bronchiectasis has used this index; therefore, limited comparison of this study with other studies is possible. The effects of the oscillatory PEP technique on secretion removal are attributed to a change in mucus rheology, 32,33 whereas the effects of the thoracic compression technique are attributed to an increase of the expiratory flow. ${ }^{34}$ Both interventions increased secretion production compared with the control sessions, but only results obtained by using the oscillatory PEP technique, with higher dry and total weight of expectorated secretions, were statistically significant. Only 3 subjects expectorated secretions in the control session, whereas 14 other subjects in the treatment group expectorated, which corroborated the benefits of oscillatory PEP in bronchiectasis. An evaluation based on the weight or total volume of secretions may affect the results because there is no distinction made between the amount of saliva and respiratory secretions in the sample collected. To reduce this bias, we evaluated the dry weight of the sputum to eliminate the effects of saliva and make the results more reliable.

In agreement with our results, another study also reported a higher volume of expectorated secretions after oscillatory PEP treatment compared with sham oscillatory 


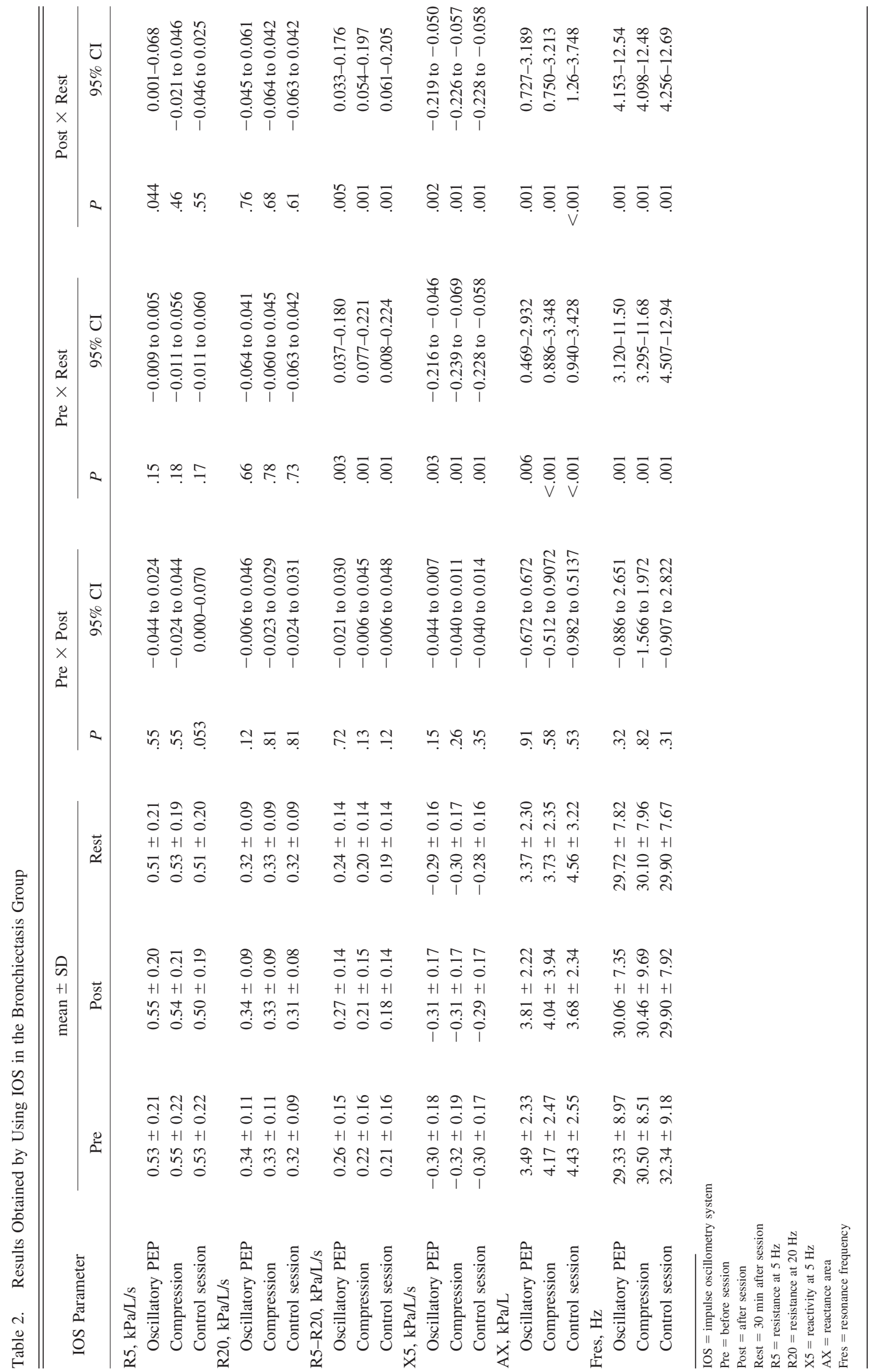




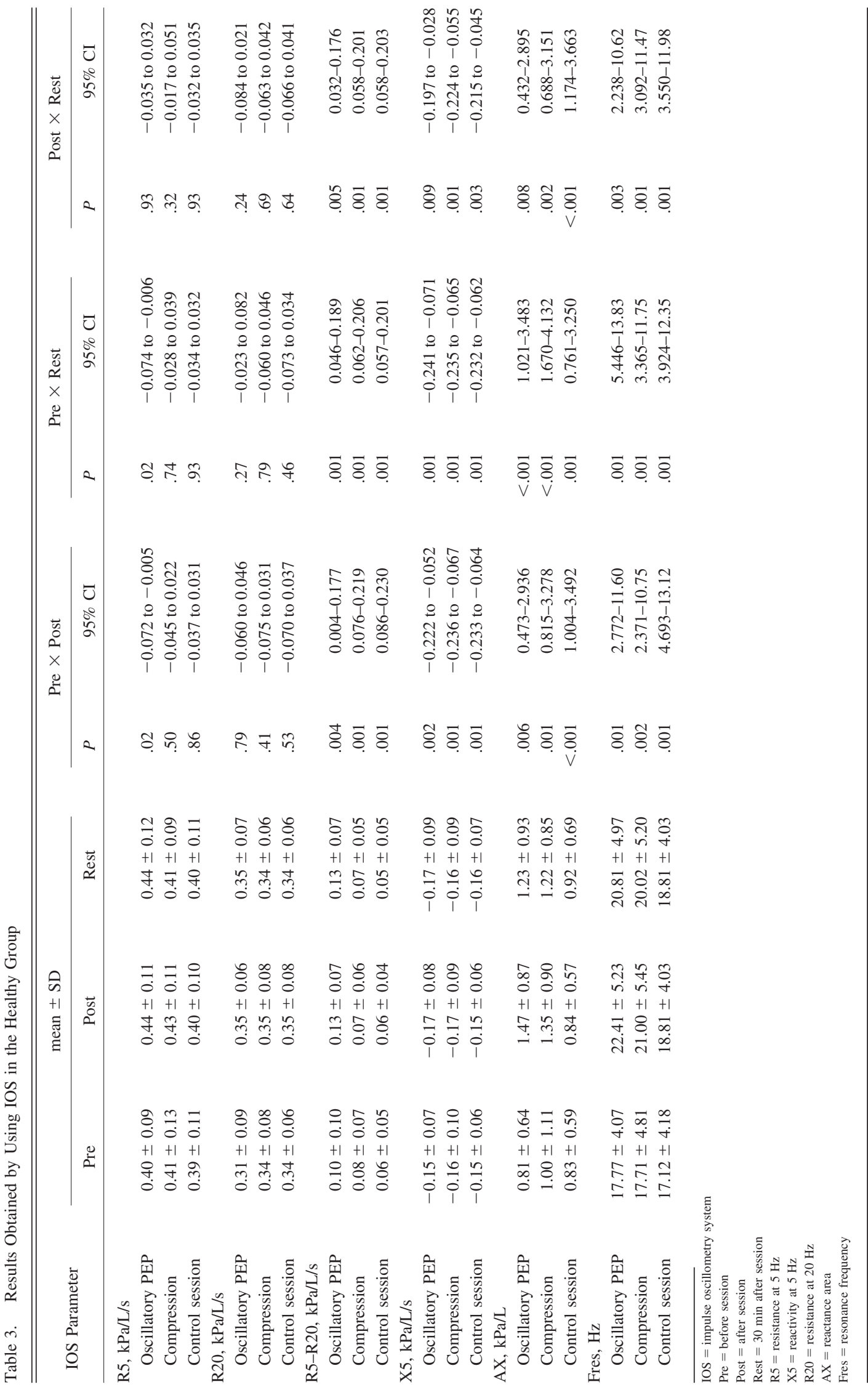




\section{Oscillatory PEP and Thoracic COMPREsSion IN Non-CF BronchiECTASIS}

Table 4. Frequency of Increased Acceptability and Tolerance Scores Reported by the Subjects

\begin{tabular}{|c|c|c|c|c|c|}
\hline Parameter & $\begin{array}{l}\text { Utility (\%) } \\
\text { (scores 1-2) }\end{array}$ & $\begin{array}{l}\text { Instructions (\%) } \\
\text { (scores 1-2) }\end{array}$ & $\begin{array}{l}\text { Achievement }(\%) \\
\quad(\text { scores 1-2) }\end{array}$ & $\begin{array}{l}\text { Exhaustion }(\%) \\
\text { (scores 6-7) }\end{array}$ & $\begin{array}{l}\text { Discomfort } \\
\text { (scores 6- }\end{array}$ \\
\hline \multicolumn{6}{|l|}{ Bronchiectasis group } \\
\hline Oscillatory PEP & 85 & 80 & 80 & 40 & 70 \\
\hline Chest compression & 80 & 85 & 75 & 80 & 70 \\
\hline Control session & 67 & 72 & 94 & 72 & 94 \\
\hline \multicolumn{6}{|l|}{ Control group } \\
\hline Oscillatory PEP & 75 & 95 & 80 & 85 & 80 \\
\hline Chest compression & 80 & 90 & 95 & 90 & 100 \\
\hline Control session & 70 & 90 & 95 & 90 & 95 \\
\hline
\end{tabular}

Volunteers' perception of the procedure's utility (Utility), ease of understanding instructions (Instructions), ease of performing the exercises (Achievement), the degree of fatigue (Exhaustion), and discomfort (Discomfort) on a scale of 1 to 7 (1, extremely, and 7, none); the best scores for Utility, Instructions, and Achievement were 1-2, and, for exhaustion and discomfort, were 6-7.

PEP. ${ }^{21}$ However, the highest volume of expectorated secretions was obtained after slow expiration with the glottis open in a lateral posture in comparison with the oscillatory PEP and control with the treatments. ${ }^{35}$ Notably, a previous report $^{35}$ and this present study applied the oscillatory PEP technique with the subject in a seated position with exhalation at tidal volume, whereas slow expiration with the glottis open in a lateral posture was performed in a position in which the subject lies on his or her side with exhalation at a volume close to the residual volume. In addition, this study used the oscillatory PEP technique for only $15 \mathrm{~min}$, whereas it was performed for $30 \mathrm{~min}$ in the present study.

We did not find any studies that evaluated the efficacy of thoracic compression on secretion removal in subjects with bronchiectasis. Our results showed comparable effects on the peripheral parameters in the subjects with bronchiectasis. Although secretion volume after compression was slightly higher compared with control sessions, it was not statistically significant when compared with the volumes obtained during either the oscillatory PEP or the control sessions.

Bronchiectasis is characterized by a cycle of bronchial inflammation and destruction that causes changes in pulmonary function with obstructive characteristics and airflow limitation. ${ }^{2}$ Impulse oscillometry is an alternative method for evaluating the resistance of the respiratory system. This technique is more sensitive for identifying alterations in the peripheral airways of patients with chronic respiratory diseases when compared with spirometry, ${ }^{36}$ and it may be a promising tool for evaluating the effects of respiratory physiotherapy. In the bronchiectasis group, there was an increase in total resistance after the control session, but a decrease after oscillatory PEP. However, resistance increased in the healthy group post flutter, which implies the technique has a favorable outcome in individuals with secretions, but may produce adverse effects in those who do not. It is important to note that the flutter is not recommended for healthy individuals, but the main lesson here 1) consideration of the potentially negative effects of incorrect application of the device and use only in specific patient populations; 2) reinforcement that the effects on airway resistance are related to secretion retention; and 3) clinical effectiveness of the oscillatory PEP is most likely disease dependent, and this device needs to be precisely indicated.

Impulse oscillometry was recently used in 2 similar studies that assessed physical therapy. ${ }^{21,22}$ In a group of 8 subjects with bronchiectasis, Figueiredo et $\mathrm{al}^{21}$ reported improvement in total resistance and the reactance area after oscillatory PEP sessions. It is worth mentioning that the group in this study had a sputum volume of approximately $50 \mathrm{~mL} / \mathrm{d}$, and although we did not evaluate this variable in our subjects with bronchiectasis, the amount of expectorated secretions during the interventions was lower than this value. ${ }^{21}$ The volume of secretions in our study increased after oscillatory PEP, with a decrease in total and peripheral resistance after both oscillatory PEP and thoracic compression. Another study on subjects with COPD and small volumes of secretion showed decreased resistance, reactance, and expiratory flow limitation after $30 \mathrm{~min}$ of oscillatory PEP, which suggested that the difference in our results could be attributed to the mechanism of the disease. $^{22}$

We found that resistances did not decrease immediately but only after $30 \mathrm{~min}$, similar to results described in subjects with COPD who presented with increased airway resistance in the immediate evaluation and a decrease observed after 20 min. ${ }^{22}$ We speculate that this effect could be attributed to episodes of coughing and mobilization of secretions, induced by the technique performed without previous use of a bronchodilator. Based on a similar hypothesis to the present study, Guan et $\mathrm{al}^{37}$ studied the resistance of the respiratory system in subjects in stable phase of bronchiectasis during exacerbation, which is a period with increased secretion production. Contrary to 


\section{Oscillatory PEP AND Thoracic COMPRESSION IN Non-CF BRONCHIECTASIS}

expectations, impulse oscillometry did not present any differences in the 3 periods studied. A possible explanation for these differences to the results in the literature was that the mechanisms that lead to obstruction in bronchiectasis have structural characteristics that do not vary with the amount of secretion.

The effects of the thoracic compression technique are not well defined, and, to our knowledge, there is no study on individuals with bronchiectasis that assessed the effects on respiratory system resistance. The abrupt application of compression can lead to bronchial collapse in patients with COPD, as evaluated by peak flow measurements. ${ }^{37}$ Therefore, in our study, compression was performed slowly, which produced no worsening of total resistance, and decreasing in the peripheral resistance, which indicated that the technique was acceptable for these individuals. The rationale by which the application of force to the chest wall works is to change the intrapleural pressure, which is transmitted through the thoracic cage, to increase expiratory flow and assist in dislodging secretions. ${ }^{38}$

Vibration techniques, active cycle of breathing, and postural drainage are all well tolerated by patients according to acceptability and tolerance scale scores. ${ }^{28}$ Our results showed that the oscillatory PEP and thoracic compression techniques were well accepted by the subjects with bronchiectasis. We evaluated the techniques in only one intervention, but, when taking into consideration the possibility of a long-term study, the high level of acceptability of the techniques was favorable for treatment adherence. For the exhaustion item, the subjects with bronchiectasis scored only $40 \%$ of the best oscillatory PEP scores, and we speculate that it may be related to the use of an active technique and/or increased cough and secretion clearance during the oscillatory PEP session. In addition, no undesirable effects, such as falling saturation or altered heart rate during the sessions, were observed, which indicated that the techniques can be used safely.

One of the main limitations of our study was related to the use of a manual technique, which was subjected to different application protocols. Although the technique was always applied by the same therapist (LHSS), the force applied to the subjects' thorax was not evaluated. Moreover, the criteria for ensuring the stability of the individuals during the protocol were only clinical, with no new complementary examinations.

\section{Conclusions}

The results suggested that the oscillatory PEP was effective for removal of secretions and for decreasing total and peripheral respiratory system resistance; thoracic compression had comparable positive effects on peripheral resistance. Both techniques were safe and well tolerated by the subjects with bronchiectasis. An increase in the respiratory system resistance in the healthy subjects after oscillatory PEP suggested that this technique must be precisely prescribed.

\section{ACKNOWLEDGMENTS}

The authors wish to thank the São Paulo Research Foundation (FAPESP) 2013/26433-0 process, and National Council for Scientific and Technological Development (CNPq).

This study was financed in part by the Coordenação de Aperfeiçoamento de Pessoal de Nível Superior - Brasil (CAPES).

\section{REFERENCES}

1. Fahy JV, Schuster A, Ueki I, Boushey HA, Nadel JA. Mucus hypersecretion in bronchiectasis. The role of neutrophil proteases. Am Rev Respir Dis 1992;146(6):1430-1433.

2. Moreira JS, Porto NS, Peixoto JJ, Felicetti JC, Cardoso PFG, Moreira ALS, Andrade CF. Bronchiectasis: diagnostic and therapeutic features. J Pneumol 2003;29(5):259-263.

3. Bergin DA, Hurley K, Mehta A, Cox S, Ryan D, O'Neill SJ, et al. Airway inflammatory markers in individuals with cystic fibrosis and non-cystic fibrosis bronchiectasis. J Inflamm Res 2013;6(Can't get issue from record):1-11.

4. Richman-Eisenstat JB, Jorens PG, Hébert CA, Ueki I, Nadel JA. Interleukin-8: an important chemoattractant in sputum of patients with chronic inflammatory airway diseases. Am J Physiol 1993; 264(4 Pt 1):L413-L418.

5. Silva JR, Jones JA, Cole PJ, Poulter LW. The immunological component of the scelular inflamatory infiltrate in bronchiectasis. Thorax 1989;44(8):668-673.

6. Jamnik S, Santoro IL, Maldonado MTM, Santos LA, Oliveira-Júnior IS. General characteristics of a sample of bronchiectasis patients followed in a respiratory clinical setting. Pneumología y Cirugía de Tórax 2002;61:5-9.

7. Chalmers JD, Hill AT. Mechanisms of immune dysfunction and bacterial persistence in non-cystic fibrosis bronchiectasis. Mol Immunol 2013;55(1):27-34.

8. Thompson CS, Harrison S, Ashley J, Day K, Smith DL. Randomized crossover study of the flutter device and the active cycle of breathing technique in non-cystic fibrosis bronchiectasis. Thorax 2002;57(5): 446-448.

9. Lee AL, Burge AT, Holland AE. Airway clearance techniques for bronchiectasis. Cochrane Database Syst Rev 2015;23(11):CD008351.

10. Hess DR. The evidence for secretion clearance techniques. Respir Care 2001;46(11):1276-1293.

11. Silva AR, Fluhr SA, Bezerra Ade L, Correia Júnior MA, França EÉ, Andrade FM. Expiratory peak flow and respiratory system resistance in mechanically ventilated patients undergoing two different forms of manually assisted cough. Rev Bras Ter Intensiva 2012;24(1):58-63.

12. Martí JD, Li Bassi G, Rigol M, Saucedo L, Ranzani OT, Esperatti M, et al. Effects of manual rib cage compressions on expiratory flow and mucus clearance during mechanical ventilation. Crit Care Med 2013; 41(3):850-856.

13. McCarren B, Alison JA, Herbert RD. Manual vibration increases expiratory flow rate via increased intrapleural pressure in healthy adults: an experimental study. Aust J Physiother 2006;52(4):267271.

14. Sivasothy P, Brown L, Smith IE, Shneerson JM. Effect of manually assisted cough and mechanical insufflation on cough flow of normal subjects, patients with chronic obstructive pulmonary disease (COPD), and patients with respiratory muscle weakness. Thorax 2001;56(6):438-444. 


\section{Oscillatory PEP AND Thoracic COMPRESSION IN Non-CF BRONCHIECTASIS}

15. Lindemann $\mathrm{H}$. [The value of physical therapy with VRP 1-Desitin ("Flutter")]. Pneumologie 1992;46(12):626-630.

16. Farbotko K, Wilson C, Watter P, MacDonald J. Change in physiotherapy management of children with cystic fibrosis in a large urban hospital. Physiother Theory Pract 2005;21(1):13-21.

17. Bhowmik A, Chahal K, Austin G, Chakravorty I. Improving mucociliary clearance in chronic obstructive pulmonary disease. Respir Med 2009;103(4):496-502.

18. Physiotherapy for people with cystic fibrosis: from infant to adult. 2009; 12 e 22. www.ecfs.eu/ipg_cf/booklet. Accessed August 20, 2018

19. Althaus P, Bovay F, Cao P, Escoffey AM, Ruiz N, Montulet F, et al. The bronchial hygiene assisted by the flutter VRP1 (module regulator of a positive pressure oscillation on expiration). Eur Resp J 1989;2(S8):693

20. Alves LA, Pitta F, Brunetto AF. Performance analysis of the Flutter VRP1 under different flows and angles. Respir Care 2008:53(3):316-23.

21. Figueiredo PH, Zin WA, Guimarães FS. Flutter valve improves respiratory mechanics and sputum production in patients with bronchiectasis. Physiother Res Int 2012;17(1):12-20.

22. Gastaldi AC, Paredi P, Talwar A, Meah S, Barnes PJ, Usmani OS. Oscillating positive expiratory pressure on respiratory resistance in chronic obstructive pulmonary disease with a small amount of secretion: a randomized clinical trial. Medicine (Baltimore) 2015; 94(42):e1845

23. Chalmers JD, Goeminne P, Aliberti S, McDonnell MJ, Lonni S, Davidson J, et al. The bronchiectasis severity index. An international derivation and validation study. Am J Respir Crit Care Med 2014; 189(5):576-85.

24. Kovelis D, Segretti NO, Probst VS, Lareau SC, Brunetto AF, Pitta F. Validation of the Modified Pulmonary Functional Status and Dyspnea Questionnaire and the Medical Research Council scale for use in Brazilian patients with chronic obstructive pulmonary disease. J Bras Pneumol 2008;34(12):1008-1018.

25. Burioka N, Sugimoto Y, Suyama H, Hori S, Chikumi H, Sasaki T. Clinical efficacy of the flutter device for airway mucus clearance in patients with diffuse panbronchiolitis. Respirology 1998;3(3):183-6.

26. Lopez-Vidriero MT. Biochemical basis of physical properties of respiratory tract secretions. Eur J Respir Dis Suppl 1987;71:130-135.

27. Murray MP, Pentland JL, Turnbull K MacQuarrie S, Hill AT. Sputum color: a useful clinical tool in non-cystic fibrosis bronchiectasis. Eur Respir J 2009;34(2):361-364.
28. Dionísio GH, Dos Santos DO, Perossi L, de Paula MH, de Souza HCD, Gastaldi AC. The influence of different mouthpieces on impulse oscillometry results. Respir Care 2018;63(5):565-572.

29. Oostveen E, MacLeod D, Lorino H, Farré R, Hantos Z, Desager K, Marchal F. The forced oscillation technique in clinical practice: methodology, recommendations and future developments. Eur Respir J 2003;22(6):1026-41.

30. Bashier B, Salvi S. Measuring lung function using sound waves: role of the forced oscillation technique and impulse oscillometry system. Breathe (Sheff) 2015;11(1):57-65.

31. Eaton T, Young P, Zeng I, Kolbe J. A randomized evaluation of the acute efficacy, acceptability and tolerability of flutter and active cycle of breathing with and without postural drainage in non-cystic fibrosis bronchiectasis. Chron Respir Dis 2007;4(1):23-30.

32. Ramos EM, Ramos D, Iyomasa DM, Moreira GL, Melegati KC, Vanderlei LC, et al. Influence that oscillating positive expiratory pressure using predetermined expiratory pressures has on the viscosity and transportability of sputum in patients with bronchiectasis. J Bras Pneumol 2009;35(12):1190-1197.

33. Tambascio J, de Sousa LT, Lisboa RM, Passarelli Rde C, de Souza HC, Gastaldi AC. The influence of FlutterVRP1 components on mucus transport of patients with bronchiectasis. Respir Med 2011; 105(9):1316-21.

34. Nozoe M, Mase K, Ogino T, Murakami S, Takashima S, Domen K. Effects of chest wall compression on expiratory flow rates in patients with chronic obstructive pulmonary disease. Braz J Phys Ther 2016; 20(2):158-165

35. Guimarães FS, Moço VJ, Menezes SL, Dias CM, Salles RE, Lopes AJ. Effects of ELTGOL and Flutter VRP1 on the dynamic and static pulmonary volumes and on the secretion clearance of patients with bronchiectasis. Rev Bras Fisioter 2012;16(2):108-113.

36. Paredi P, Goldman M, Alamen A, Ausin P, Usmani OS, Pride NB, Barnes PJ. Comparison of inspiratory and expiratory resistance and reactance in patients with asthma and chronic obstructive pulmonary disease. Thorax 2010;65(3):263-267.

37. Guan WJ, Gao YH, Xu G, Lin ZY, Tang Y, Li HM, et al. Impulse oscillometry in adults with bronchiectasis. Ann Am Thorac Soc 2015; 12(5):657-665

38. Lee AL, Button BM, Tannenbaum EL. Airway-clearance techniques in children and adolescents with chronic suppurative lung disease and bronchiectasis. Front Pediatr 2017;24:5:2. 